\title{
IMPLICANCIAS BIOÉTICO-LEGISLATIVAS DEL CONSENTIMIENTO INFORMADO EN LA REDACCIÓN DEL PROTOCOLO DE ABORTO TERAPEUTICO
}

\author{
Legislative bioethical implications of Informed Consent in the Drafting \\ of the Therapeutic Abortion Protocol
}

Belú Fiorella Pérez Dávila*

Recepción: 02-10-2018

Aceptación: 31-10-2018

\begin{abstract}
RESUMEN
El aborto terapéutico es una práctica médica que se encuentra despenalizada en nuestro país, y está regulada bajo un procedimiento estandarizado a nivel nacional en el comúnmente denominado Protocolo de Aborto Terapéutico (PAT), previo Consentimiento Informado (CI) de la gestante y sin incluir los derechos de su hijo. El presente artículo contiene un análisis bioético, desde las bioéticas personalista y principialista, así como legislativo del CI de la gestante regulado en el PAT, como requisito para la realización de un aborto por razones terapéuticas. Con lo cual conoceremos su grado de idoneidad, tanto desde el enfoque bioético como el legal.
\end{abstract}

\section{Palabras clave}

Consentimiento Informado, aborto terapéutico, bioética personalista, bioética principialista, legislación, protocolo médico.

\begin{abstract}
Therapeutic abortion is a medical practice that has been decriminalized in our country and is currently regulated under a standardized procedure at the national level in the so-called Protocol of Therapeutic Abortion (PAT), requiring the informed consent (IC) of the pregnant woman but without considering the rights of the child. The present paper contains both a bioethical analysis, from a principialistic and personalistic perspective, and a legislative analysis of the IC of the pregnant woman regulated by the PAT as a requirement for the realization of an abortion for therapeutic reasons. It seeks to discern its degree of suitability, both from a bioethical and a legal standpoint.
\end{abstract}

\section{Keywords:}

Informed consent, therapeutic abortion, personalistic bioethics, principialistic bioethics, legislation, medical protocol.

* Docente de la Universidad Marcelino Champagnat: biojuridicayetica@gmail.com 


\section{INTRODUCCIÓN}

En el presente artículo analizamos el contenido bioético y legislativo del Consentimiento Informado de la madre gestante para la realización de un aborto justificado por razones terapéuticas, regulado en la "Guía Técnica Nacional para la estandarización del procedimiento de la Atención Integral de la gestante en la Interrupción Voluntaria por indicación Terapéutica del Embarazo menor de 22 semanas con consentimiento informado, en el marco de lo dispuesto en el artículo $119^{\circ}$ del Código Penal”, o comúnmente denominado Protocolo de Aborto Terapéutico (PAT), promulgado mediante Resolución Ministerial N 486-2014/MINSA.

Dicho PAT compromete a tres personas: la vida en desarrollo intrauterino, que es eliminada con el aborto terapéutico, la madre gestante, cuyo $\mathrm{CI}$ es crucial para la prosecución de esta clase de aborto, y el médico, quien se encuentra obligado a recabar dicho CI previamente a la ejecución del aborto terapéutico. Dada esta dinámica, hemos evaluado el CI regulado en el PAT, a fin de conocer si su orientación bioética es acorde con el respeto por las personas implicadas, y si legislativamente cumple con las garantías que amparan a la madre gestante con respecto al debido ejercicio del derecho al $\mathrm{CI}$, frente a los actos de los profesionales de la salud que no obren conforme a la "lex artis" de su profesión, teniendo en cuenta la salud física y mental de la madre ante los riesgos que sobrevienen a la intervención quirúrgica abortiva. Por estos motivos hemos formulado el siguiente cuestionamiento: ¿Qué implicancias bioéticas y legislativas tiene para la madre gestante la aplicación del CI en la redacción del PAT?
Para responder a esta pregunta, desarrollaremos los aspectos relevantes de la teoría del CI desde el enfoque del paciente destinatario de las terapias médicas, al igual que su componente bioético, desde el personalismo y el principialismo, identificando la bioética a la cual se orienta según lo regulado por el PAT. Asimismo, analizaremos el CI de la madre gestante en la redacción del PAT y su coherencia con el resto de la legislación referente al $\mathrm{CI}$.

\section{ASPECTOS RELEVANTES DEL CONSENTIMIENTO INFORMADO}

El CI es el derecho indispensable que tiene todo paciente a ser informado sobre los riesgos que se encuentren vinculados a una intervención médica, con el fin de que pueda brindar o denegar su consentimiento (Martín, 2005, pp. 147 - 148). Dicha información ha de ser adecuada y suficiente; sobre el tratamiento, sus beneficios y sus riesgos, no bastando el proporcionarla, sino el asegurarse de su comprensión (Vizcaya et al, 2014, p. 9). ${ }^{1}$ Para esto, el paciente debe contar con la capacidad necesaria, ${ }^{2}$ a fin de evaluar su situación y tomar una decisión, asumiendo las consecuencias de la misma (Rubio, 2005, pp. 133-134). En consecuencia, el CI resulta de un diálogo entre el profesional de la salud y el paciente, en donde este recibe la información que requiere para tomar una decisión sobre su salud, realizando las consultas pertinentes, y el profesional las absuelve, asegurándose de que la información ha sido comprendida.

Una vez recibida la información, el paciente debe decidir otorgar o no su CI, asumiendo los riesgos y consecuencias

1 Otros autores sostienen que, al ser el derecho de todo paciente, independientemente de su capacidad, se le ha de informar de acuerdo a su posibilidad de comprender (cf. Civeira, 2010, p. 25).

2 La capacidad implica tanto la edad del paciente, como el grado de entendimiento que posea al momento de proporcionar su $\mathrm{CI}$. 
que previamente ha comprendido. Aunque parezca evidente, es adecuado resaltar que su decisión debe ser voluntaria, libre de coerción y manipulación. La misma que, cuando se trate de actuaciones médicas relevantes, ha de quedar documentada, como prueba de su realización (Novoa, 2013, pp. 22 - 23). Puesto que, habiendo entendido la información, el paciente podría estar en desacuerdo con su médico y aun así firmar el CI por insistencia o chantaje del profesional.

De otro lado, el CI, como todo derecho, tiene límites que establecen las fronteras sobre lo que es posible decidir y ejercer la autonomía personal. Uno de ellos consiste en que su licitud depende de la disponibilidad del objeto sobre el cual recae la decisión (Ossadón, 2012, p. 326). De este modo, el paciente podrá autorizar procedimientos que involucren únicamente su salud, permitan su recuperación e impliquen un riesgo razonable. Otro, es el que se origina en el derecho al ejercicio legítimo de una autoridad, oficio u cargo. Así, el médico deberá actuar conforme a la lex artis de su profesión y podrá evitar consentimientos que contravengan a su ética profesional (Ossadón, 2012, pp. 335 - 337).

\section{IMPLICANCIAS BIOÉTICAS DEL CONSENTIMIENTO INFORMADO REGULADO EN EL PAT}

El CI expresa el respeto y la consulta al paciente, integrando su sistema de creencias con el tratamiento de lo que afecta a su corporeidad. En este apartado, desarrollaremos el CI desde los dos modelos de bioética más representativos: personalismo y principialismo, a fin de averiguar el modo de comprender dicho derecho, desde dichos modelos, y la visión con que se encuentra regulado dentro del PAT.

\subsection{El Consentimiento Informado desde la bioética personalista}

Según la bioética personalista, el Cl de los pacientes ha de ser precedido por una reflexión ética cuya base y principio fundamental sea el respeto absoluto e incondicional del ser y la dignidad de la persona, ya que compromete a cuestiones éticas referentes a su vida (Carrasco, 2005, p. 209), bajo la comprensión de que la persona es el punto de referencia y el fin de la realidad a la cual trasciende, merecedora de una valoración plena (Sgreccia, 2007, p. 71).

En las siguientes líneas analizaremos el contenido del CI y su vinculación con la relación médico paciente para un consentimiento válido desde la bioética personalista; así como sus principios. Esto nos permitirá identificar los aspectos de este modelo de bioética observables en la redacción del PAT, así como los aspectos en los que difiere.

El Consentimiento Informado y la relación médico paciente desde la bioética personalista

La naturaleza de la relación médico paciente consiste en un pacto entre personas. Por un lado, está el paciente, principal responsable de su vida y su salud. Está consciente de su enfermedad y la amenaza que esta le supone para su autonomía, de sus límites e incompetencia para recuperar por su cuenta su salud o prevenir el daño que la enfermedad pudiere ocasionarle y por este motivo se dirige hacia otra persona: el médico. Este realiza la tarea de colaborador del paciente, haciendo uso de su preparación y experiencia médica, cuenta con la capacidad para ayudarlo (Sgreccia, 2007, p. 279). El CI se encuentra implícito a lo largo de esta relación, desde que la persona se 
presenta ante el profesional de la salud, constituyéndose en paciente. Es un deber permanente del médico el proporcionar la información correspondiente a las terapias aplicables al paciente y solicitar su respectivo consentimiento explícito las veces que sea necesario (Sgreccia, 2007, p. 223). Esto se debe a que el CI forma parte de la lex artis de la tarea profesional del médico (Gonorazky, 2015, p. 1)

En esta relación entran en juego la parte técnica y la parte humana del médico. La primera se refiere al conocimiento científico de la enfermedad. La parte humana espontánea se origina en el ejercicio de la profesión: la comunicación del médico con el enfermo y con sus amigos y familiares, para conocer su modo de vivir, al repercutir directamente en el estado de salud del enfermo (Pardo y Echarte, 2015, pp. 200-201). Con esto se comprende al paciente y se tiene en consideración sus opiniones y decisiones, que, guiadas por el consejo médico, permiten un $\mathrm{Cl}$ adecuado, el mismo que resulta constructivo para el desarrollo ético y autoconocimiento de ambos. En esta línea, "... el personalismo comprende y asume que el hombre se hace hombre sólo frente al hombre, se hace yo - sujeto frente al tú - sujeto, no frente al tú - objeto" (Burgos, 2013, p. 24). Ambas partes tienen el deber de actuar éticamente, ejerciendo valores de fidelidad mutua, ya que son personas (Pardo y Echarte, 2015, p. 208), incluyendo para su actuación tanto la subjetividad de la persona, como la realidad desde la cual percibe y discierne, y no cerrarse a considerar únicamente la parte subjetiva de la persona, suprimiendo la noción de la realidad del acto en concreto (Pardo, 2012, p. 4).

El PAT regula sobre los embarazos cuya complicación puede poner en riesgo la vida o la salud de la madre gestante. Las personas implicadas son tres. Dos de ellas son pacientes, por cuyas vidas se deben desplegar los esfuerzos médicos, estos son la madre gestante y el hijo intrauterino (Besio, 2013 , pp. 53 - 55), por lo que se debe hablar de dos relaciones médico paciente: la que existe entre el médico y la madre gestante y la relación entre el médico y el hijo de la misma. De ello surge el deber del médico de colaborar con la recuperación de la salud de la madre y de su hijo. Siendo que el hijo no tiene la capacidad para decidir sobre las terapias que repercutirán sobre su vida y su salud, sus padres, como representantes suyos, deben tomar las decisiones que le favorezcan más. El PAT no tiene en cuenta esta acotación, subsumiendo al hijo en la madre y suprimiendo los derechos del primero.

\section{El Consentimiento Informado regulado en el Protocolo de Aborto Terapéutico desde los principios de la bioética personalista}

El CI es el resultado del respeto que se le debe al paciente, en su dignidad personal, ratificada en toda la fundamentación filosófica de la bioética personalista. Permite que el paciente sea informado oportunamente y pueda tomar las decisiones sobre las terapias que le serán aplicadas, las mismas que deben estar en armonía con su dignidad.

En las líneas sucesivas, basándonos en los principios de la bioética personalista: defensa de la vida física, libertad y responsabilidad, totalidad o principio terapéutico, y sociabilidad y subsidiaridad, analizaremos si la regulación del PAT ha coincidido con alguno.

3 Cabe acotar que el art. III del Título Preliminar de la Ley General de Salud, 1997, reconoce que el concebido es sujeto de derecho en el campo de la salud. 
Principio de libertad y responsabilidad: $\mathrm{El} \mathrm{CI} \mathrm{se} \mathrm{explica} \mathrm{mediante}$ este principio, que tiene en cuenta dos cuestiones: la libertad y la responsabilidad. El paciente debe encaminar su libertad hacia la recuperación de su salud, lo cual requiere de una reflexión ética, justificada y respetuosa, ya que, de lo contrario, bastaría la voluntad del paciente (Sgreccia, 2007, p. 232) con independencia del logro de su auténtico bien; en esta medida la eutanasia sería igual de respetable que cualquier procedimiento médico (Sgreccia, 2007, p. 232). Por esto, todo Cly decisión del paciente deberán contener y respetar los elementos para la valoración del acto humano. Para determinar su moralidad se debe considerar los elementos que normalmente se estudian del acto moral: objeto, fin y circunstancias (Pardo, 2008, p. 106).

Por su parte la responsabilidad tiene dos aspectos: el primero consiste en la conciencia personal de la valoración moral de las acciones, sabiendo que le pertenecen a quien las realiza y se deben a él; el segundo, radica en el deber exclusivo, que tiene la persona que las realiza, de responder por sus consecuencias (Fuentes, 2006, p. 54). Por la responsabilidad, el paciente está obligado a cuidar de su vida y la ajena, debiendo colaborar con el médico en los cuidados necesarios para salvaguardarla (Sgreccia, 2007, p. 222).

\section{Principio de defensa de la vida} física: Este principio constituye, no sólo el principal límite al CI del paciente también es el que prima entre todos los principios de la bioética personalista. Y se debe a que la vida es un valor fundamental y por encima de este sólo está el bien total y espiritual de la persona (Sgreccia, 2007, pp. 219 - 222). Este principio hace referencia a lo comprendido como vida orgánica, cuerpo físico. El cuerpo es coesencial para la existencia, la persona ingresa en el tiempo y en el espacio, se manifiesta y realiza mediante un cuerpo orgánico. Su conservación y dinámica se logran a través de la vida física (Sgreccia, 2007, pp. 218). El PAT no tiene en cuenta este principio, ya que sólo tiene en cuenta la vida de la madre gestante y no la de su hijo, quien es la víctima del aborto terapéutico consentido por su madre.

\section{Principio de totalidad o principio} terapéutico: Se comprende que el cuerpo humano es un todo. El conjunto de cada una de sus partes comporta la unidad de la realidad personal (Sgreccia, 2007, p. 223). El daño que pueda sufrir cualquier parte del cuerpo, como un brazo o una pierna, afectará a toda la persona, siendo posible sacrificar a la parte para salvar a toda la persona (Fuentes, 2006, p. 61).

De otro lado, lo terapéutico de este principio implica los deberes derivados de cuidar la vida humana (Serrano, 1993, p. 21). Para su aplicación hace falta tener en cuenta determinadas condiciones: "1) que se trate de una intervención sobre la parte enferma o la que es causa directa del mal, para salvar al organismo sano; 2) que no existan otras maneras o medios para evitar la enfermedad; 3) que haya una buena posibilidad, proporcionalmente alta, de éxito; 4) y que se dé el consentimiento del paciente" (Sgreccia, 2007, p. 224; García, 2013, p. 68).

El CI regulado por el PAT para la realización del aborto es contrario a este principio; ya que la intervención recae sobre otro ser, eliminándolo por completo, el mismo que es independiente de la mujer gestante, no siendo una parte suya.

Principio de sociabilidad y subsidiariedad: Desde la sociabilidad de este principio, se reconoce a la persona 
como el centro de la sociedad, la misma que valora sus aportes y le corresponde mediante las diversas clases de apoyo para su realización (García, 2013, p. 68).

Desde la subsidiaridad, la sociedad se solidariza con las personas que por sí mismas no pueden proporcionar aporte alguno, los más necesitados (García, 2013, p. 227). En el PAT no se cumple este principio, ya que esta norma ha omitido el debido respeto y valoración de la vida humana en desarrollo gestacional, condición mínima para la existencia. Asimismo, no existe la solidaridad con la madre gestante, ya que no se le presenta otra opción que el aborto.

\subsection{Análisis del Consentimiento Informado desde la bioética principialista}

El principialismo es una teoría que se basa en cuatro principios, que son la manifestación de los valores más aceptados por el consenso social (Martín, 2005, p. 21). Este modelo de bioética encuentra su significado en un "sistema" y desconoce el sentido filosófico, asociado a la universalidad, de la palabra que le da su denominación: principios (Requena, 2005, pp. 47-50). A decir de Engelhardt, uno de sus autores de referencia: "Existe una dificultad fundamental para establecer la objetividad de toda preferencia moral o de las evaluaciones en general. Se necesita una pauta o norma con referencia a la cual sea posible ordenar los resultados..." (Engelhardt, 1995, p. 60). Con el sutil rechazo de la objetividad, importante para la universalidad de las normas, nos permite entender que recurre a otros criterios para conseguir las pautas que menciona: a través de la clasificación de los resultados emergentes de la casuística según su rango de importancia y armonizando los intereses en juego (Engelhardt, 1995, p. 60). El CI de los pacientes debe basarse, ya no en principios universales intrínsecos a la persona humana, sino en principios conformes con el consenso de la sociedad en la que se tenga que tomar la decisión bioética. En las siguientes líneas, analizaremos el CI en la relación médico paciente, así como los principios de este modelo de bioética.

\section{El Consentimiento Informado y la relación médico paciente desde la bioética principialista}

El trasfondo de la visión principialista de la relación médico paciente pasa por comprender al hombre como un ser eminentemente egoísta, razón por la cual hace falta una norma que controle sus excesos. Ya sea para que no sea muerto por otro hombre (Hobbes), poder gozar de las comodidades de la vida a través de la relación comercial (Locke), o para tener la libertad de vivir conforme con nuestros impulsos (Rousseau), para evitar el engaño y regular las relaciones sociales, las mismas que no son comprendidas como emergentes de nuestra naturaleza humana, sino como un concierto interesado en el bienestar individual que llevará de modo indirecto a un bienestar social. Desde este enfoque, el CI debería desarrollarse en una relación médico paciente de carácter mercantil, en la que el paciente se encuentra interesado en recuperar su salud y el médico en recibir una retribución económica por sus servicios profesionales. De ahí se entiende la gama de alternativas de tratamiento que el médico se encuentra obligado de informar al paciente, a modo que este pueda escoger el de su preferencia (Pardo y Echarte, 2015, pp. 202 203; Bernar, 2000, p. 79).

El PAT no reconoce como paciente a la mujer embarazada desde el inicio 
de la relación con su médico, sino que la denomina como "gestante". Sólo empieza a mencionársele como tal a partir de la solicitud que realiza para un aborto (art. 6 inc. 3.2, art. 6, inc. 8; art. 6 inc. 9). Asimismo, se le vuelve a tratar como "gestante" cuando decide revocar su consentimiento (art. 6 inc. 6, párrafo 5). Ya que una gestante no necesariamente será una paciente, pero sí hay casos en los que la paciente es una mujer que se encuentra en un periodo de gestación, el término "gestante" resulta impreciso, al momento de verla como la destinataria de todos los derechos que le corresponderían si fuese observada como una paciente. El PAT reduce sus derechos sanitarios al momento en que la mujer solicita el aborto, siendo afín con el aspecto contractual de este modelo de bioética. Con esto, la mujer que asumiera los riesgos gestacionales derivados de su decisión de continuar con su embarazo quedaría desprotegida.

\section{El Consentimiento Informado regulado en el Protocolo de Aborto Terapéutico desde los principios de la bioética principialista}

La bioética principialista, ha propuesto cuatro principios: autonomía, beneficencia, no maleficencia y justicia. Desde esta bioética, el CI de los pacientes sería válido siempre que cumpla con los mismos, teniendo en cuenta sus límites.

Principio de autonomía: Este principio se deriva del principio del 'respeto de las personas' propuesto por Beauchamp y Childress, el mismo que tenía en cuenta, por una parte, la exigencia de la autonomía de los individuos y, por otra, la protección de las personas con una autonomía mermada (Martín, 2005, pp. 9; 11). El posterior cambio de dominación a 'principio de respeto a la autonomía' resalta la eliminación de cualquier injerencia médica en las decisiones del paciente (Requena, 2005, p. 75), con el riesgo de deteriorar la relación y comunicación natural entre el médico y el paciente.

Esta variación tiene consecuencias que colocan en desventaja al paciente, frente a su médico. Cuando nos preguntamos sobre el significado de la autonomía, podemos encontrar dos aproximaciones. Puede ser entendida como el medio para el ejercicio de la razón especulativa y la razón práctica, esenciales para el razonamiento humano, al permitir que ambas logren sus fines: la verdad y el bien, respectivamente. O puede estar basada en el rechazo de la vinculación entre la autonomía y el bien, por lo que no hay presupuestos previos que esta deba seguir, convirtiéndose en lo más importante de por sí (Sgreccia, 2007, pp. 13 - 14).

Al apartar la contribución del médico en la toma de las decisiones del paciente, advirtiendo que la situación de este es física, psicológica y emocionalmente, vulnerable, y que carece de una adecuada preparación científica, con la que sí cuenta el médico, el paciente se topa con la dificultad de identificar la decisión médica que le hace un bien verdadero. Consideramos que el principio de autonomía en el modelo de la bioética principialista se desvincula de la noción del bien del paciente, primando una autonomía temeraria.

El CI regulado en el PAT es afín a esta última autonomía; pues permite que sea la paciente quien le solicite a su médico tratante la realización del aborto (art. 6 inc. 2.2), el mismo que deberá solicitarlo de manera escrita a la Jefatura del Departamento de Gíneco - 
Obstetricia. Reforzándose cuando, ante la negativa a la petición de la paciente, se la faculta a "solicitar al Director General del establecimiento de salud que se realice una nueva Junta Médica con otros médicos (art. 6 inc. 3.4), designando la representación de dicho director al médico tratante que evaluó el caso (art. 6 inc. 3.4).

Principio de beneficencia: Este principio es tan antiguo como el criterio aplicado a lo largo de la práctica médica. Al igual que el principio de no maleficencia, se encuentra en el cuerpo hipocrático y, además, en la Declaración de Ginebra (1948), cuyo contenido norma la obligación del médico de elegir lo que crea mejor para el enfermo (Martín, 2005, pp. 12 - 13). Sin embargo, la bioética principialista lo comprende diferente, ya que consiste en un juicio general dirigido a orientar a los clínicos a resolver los muy diversos y complejos problemas referentes a las acciones humanas (Requena, 2005, p. 38), y se limita a normar la obligación de no causar daño (Martín, 2005, pp. 12 - 13).

Esto implica que el principio de beneficencia puede ser interpretado en favor del alcance del beneficio de personas distintas al paciente. De acuerdo con la metodología que emplea el principialismo la solución bioética puede ser radicalmente distinta. Dado que cuando hay dos o más personas implicadas en el problema, se emplea el criterio del consenso colectivo. Lejos de considerar los criterios objetivos de la afectación de las personas cuya vida o salud corre peligro, ante un conflicto entre los principios generales que deban aplicarse, se prioriza que haya un equilibrio entre las consecuencias que la decisión bioética pueda tener. Asimismo, al primar las consecuencias, rige otro principio denominado utilitarismo de la regla, ya que las mismas no deberán afectar a personas ajenas a la terapia o a la sociedad (Sgreccia, 2007, pp. 230 231). Esto se observa en el PAT, debido a que resulta cuestionable que un aborto beneficie a la mujer más que al sistema de salud que escatima en gastos para proteger la integridad de las mujeres y las vidas que gestan, siendo el aborto la medida más rápida de solucionar los problemas que circundan a la atención integral de la gestante.

\section{Principio de no maleficencia: posee} dos acotaciones particulares que lo alejan de su contenido originario. La primera es respecto al análisis de los beneficios emergentes de un procedimiento, ya que muchos de ellos implican un detrimento para la salud del paciente, pero se justifican según el beneficio obtenido (Ferro et al, 2009, p. 2). El inconveniente está en identificar los criterios empleados para reconocer lo que debe considerarse como beneficioso para el paciente, lo riesgoso y el método de ponderación entre ambos. La segunda acotación, no tan general entre los autores, radica en que este principio relativiza lo establecido en la ética médica sobre el no hacer daño; liberando al médico de las obligaciones contrarias a su ética profesional, con el solo deber de remitirlas a otro médico disponible (Gómez, 2009, p. 232). Con esto, la consideración ética de la acción médica depende, no de su valoración objetiva, sino de la apreciación subjetiva del médico.

De otro lado, podría haber un conflicto entre este principio con el principio de autonomía, al impedir al paciente un procedimiento inmediato. Esto es más notorio cuando varios médicos rechazan lo solicitado por el paciente, tal como se encuentra contemplado por el PAT, ya que la madre gestante podría solicitar una nueva junta médica, ante la 
denegatoria de la primera.

Principio de justicia: implica el trato igualitario a los casos que presentan igualdad de condiciones (Martín, 2005, pp. 16 - 17), que el Estado debe realizar una distribución equitativa de los recursos: servicios de salud, investigación, etc. (Sgreccia, 2007, p. 234; Martín, 2005, p. 10), y que se le ha de dar a cada uno lo que necesita, por considerarse como un requerimiento de la justicia social, para frenar las desigualdades y ayudar al menos favorecido (Ferro et al, 2009, p. 4).

El problema con este principio radica en que no hay una comprensión universal de la idea de la justicia (Martín, 2005, pp. 13 - 17). Sería aplicado en los términos que la comunidad implicada considere justos, no importando la fundamentación básica y común a toda persona de los valores en juego. Dando lugar a posibles vulneraciones de la dignidad que, basadas en la invocación de la justicia, atiendan a intereses particulares y utilitaristas (Sgreccia, 2007, p. 234). En el PAT hemos advertido la presencia de este principio, ya que, al regular un aborto directo ante la situación compleja de la madre gestante, no considera el respeto por el irrenunciable derecho a la vida de la persona en periodo de gestación. Esto, aunado a la influencia social y política por la liberación del aborto ${ }^{4}$, superpone la voluntad humana sobre la vida misma.

\section{LEGISLACIÓN SOBRE EL CONSENTIMIENTO INFORMADO PARA LA EJECUCIÓN DE UN ABORTO TERAPÉUTICO}

En las siguientes líneas, analizamos terapéutico según la redacción del PAT, en conjunto con la legislación nacional que regula dicho derecho, a fin de conocer su grado de coherencia normativa.

\subsection{Derecho de la madre gestante a la información según el PAT}

\section{Contenidos específicos de la información}

La información que recibirá la madre gestante se encuentra señalada en dos partes de la redacción del PAT. En la primera se encuentra como una indicación para el médico tratante, dentro de los procedimientos administrativos asistenciales: la de informar a la gestante cuando el embarazo ponga en riesgo su vida o causa en su salud un mal grave y permanente (art. 6 inc. 2.1). Los aspectos que se le informarán son: el diagnóstico, el pronóstico, los riesgos graves para su vida o su salud y los procedimientos terapéuticos que correspondan.

Luego, el PAT considera que la entrega de información constituye un deber para el médico que realizará el aborto terapéutico (Art 6,6). Por esto, debe comunicar a la mujer o a su representante legal, de una manera completa y detallada, la siguiente información sobre: el diagnóstico, el procedimiento abortivo, los riesgos en el caso de someterse o no a dicho procedimiento, el pronóstico para ambos casos, las alternativas del procedimiento a las que hubiera lugar y toda la información relevante para la toma de una decisión libre y razonada.

En esta parte el PAT sólo indica los aspectos sobre los cuales la mujer ha de ser informada, omitiendo la

el CI para la realización de un aborto

4 Esto se evidencia de la revisión de la bibliografía en la que se fundamenta el PAT (parte final de dicho documento), que es información publicada por ONGs que promueven los mal llamados "derechos sexuales y reproductivos" y el "aborto seguro", por poner algunos ejemplos: PROMSEX y Flora Tristán. 
exposición detallada de cada uno de ellos. Estas indicaciones informativas ya se encuentran reguladas, de manera más completa, en la Ley que establece los Derechos de las Personas Usuarias de los Servicios de Salud (Ley N²9414, 2009, Art. 15 inc. 2.f), que modificó el art. 15 de la Ley General de Salud (LGS) (Ley $\left.N^{\circ} 26842,1997\right)$, y su respectivo Reglamento (Decreto Supremo No 027 2015-SA, 2015, Art. 15). La omisión informativa en la que incurre el PAT se agudiza al incumplir los objetivos de toda Guía Técnica, documento normativo del MINSA, ${ }^{5}$ ya que debería contener la información de cada uno de los aspectos relevantes para el tratamiento de la mujer gestante, desde antes, durante y después de su decisión; tanto de realizarse un aborto denominado terapéutico como de optar por continuar con su embarazo, asumiendo los riesgos posibles.

Dada la regulación prohibitiva del aborto en nuestro país, la acotación sobre todo el proceso de atención de la madre gestante resulta indispensable. El PAT debería marcar los límites legales y éticos de esta práctica, el CI de la mujer se encuentra sujeto a su regulación, ya que, si estuviese prohibido, dicho CI no tendría lugar. En esta medida, la indicación sobre los aspectos que el médico debe informar a la mujer, antes mencionados, deberán materializarse en la redacción de todo el documento que venimos tratando como PAT; y no mostrar dichas omisiones. A continuación, evaluamos dichas indicaciones informativas.

\section{Sobre el objeto de la información:} al tratarse de una situación compleja que compromete a dos vidas humanas, la de la mujer y la de su hijo, resulta indispensable que aclaremos sobre quién versa la información antes expuesta: si es sobre la enfermedad de la mujer, o sobre el peligro que ocasiona el embarazo. El PAT hace mención al contenido de la información que se entregará, pero omite el que esta deba referirse a la enfermedad, antes bien, se enfoca en la información para someterse o no al "procedimiento" abortivo (art. 6 inc. 6), o aquella referida al embarazo riesgoso (art. 6 inc. 2), privando a la mujer gestante de la información completa sobre su actual estado de salud y promoviendo que esta se incline por el aborto. Por esto, el PAT contradice a la Ley $N^{\circ} 29414$, y su Reglamento, antes citada, que establece claramente el derecho de toda persona a ser informada sobre su enfermedad, causa por la que se interviene en perjuicio de la vida intrauterina. Por estos motivos, podemos advertir un primer sesgo informativo para el CI según el PAT

\section{Información sobre el diagnóstico:}

habiendo visto que la única información que recibe la mujer gestante versa sobre el procedimiento abortivo, el diagnóstico versa sobre las entidades clínicas, por cuya presencia se permite el aborto por razones terapéuticas. El art. 6.1 del PAT divide a dichas entidades en dos grupos: el primero consiste en una lista de 10 causales, y el segundo grupo que consiste en "cualquier otra patología materna", con lo cual incluye a diversas circunstancias médicas, sin nombrarlas y bastando la presencia de un riesgo para la vida de la gestante, o que la patología genere en su salud un mal "grave y permanente". Al no definir dicho mal, a fin de evaluar la procedencia o no de un aborto terapéutico, no establece, ningún límite objetivo para la consideración de la

5 Después de revisar otros documentos normativos de esta índole (Resolución Ministerial N 527-201 1/MINSA, 2011; Resolución Ministerial N²98-2011/MINSA, 2011; Resolución Ministerial N 1021-2010/MINSA, 2010), podemos ver que una de sus características consiste en ser detalladas, por lo que deben especificar sus procesos, teniendo en cuenta: definiciones operativas, conceptos básicos, enumeración y descripción de sus procesos, así como las conclusiones y recomendaciones sobre la utilidad de su empleo. 
práctica abortiva. Pudiendo ocurrir que el médico le recomiende a la mujer un aborto bajo consideraciones subjetivas, cuyo mal sea tolerable, y esta acceda por no saberlo. Asimismo, con esto abre la posibilidad de la ejecución de abortos injustificados terapéuticamente.

En cuanto al primer grupo, el PAT sólo proporciona los términos médicos de cada una de las entidades clínicas, sin acotar ningún tipo de descripción, ni especificación relevante para la mujer, de cada una de dichas entidades, lo cual le impide la comprensión de su problema de salud.

\section{Información sobre el procedimiento} abortivo: El contenido del procedimiento abortivo que se le debería informar detalladamente a la madre gestante antes de proporcionar su CI se encuentra en tres apartados del PAT. En el primero, regula que el médico ejecutante del aborto deberá realizarle previamente un procedimiento de evaluación (art. 6.4). Este incluye: Anamnesis: implica la revisión de su historia clínica; examen clínico, consistente en el control de las funciones vitales, exámenes del aparato respiratorio y cardiovascular, del abdomen, ginecológico, con uso del espéculo, y de tacto vaginal; exámenes auxiliares, que incluyen las pruebas serológicas, para la detección de las enfermedades de transmisión sexual, sífilis y VIH y otros exámenes para el diagnóstico de enfermedades concomitantes; y la aplicación de inmunoglobulina Anti-RH.

Si bien estas evaluaciones no supondrían riesgo biológico para la mujer, debería informársele, a fin de que cuente con una disposición adecuada en cada momento. Dado que este conjunto de pruebas implica una invasión a la intimidad de la paciente, y su desconocimiento la podría incomodar más de lo debido.
En el segundo apartado, el PAT contiene los procedimientos del momento exacto del aborto (art. 6.7). En esta parte menciona dos métodos, cuya realización depende del tiempo de gestación: en el primero abarca hasta las 12 semanas de gestación: la aspiración manual endouterina, o el uso de misoprostol. En el segundo, entre las 13 y 22 semanas de gestación: tiene en cuenta el uso del misoprostol y después de la expulsión del niño, el legrado uterino.

Un tercer apartado contiene información sobre los cuidados de la mujer posteriores al aborto terapéutico (art. 6.8.): el médico tratante debe proporcionarle las instrucciones muy claras sobre los cuidados necesarios. La paciente debe conocer que después del aborto habrá sangrado y eventualmente dolor

Observamos que esta información es bastante reducida y que, además, no es posible determinar si la mujer gestante conocía sobre el procedimiento que le correspondería antes de brindar su CI.

\section{Otros contenidos informativos:}

existen, también, otros aspectos informativos que no se encuentran nombrados, ni detallados en el PAT:

- Información sobre los riesgos del procedimiento abortivo en el PAT: pese a que regula que la mujer debe conocer sobre los riesgos tanto en el caso de someterse al aborto, como en el caso de no someterse al mismo, únicamente hace mención a la eventual presencia del dolor después del aborto, que cederá con la ingesta de analgésicos (art. 6.8.). Lamentablemente, no contiene ningún apartado que incluya los riesgos y los detalles. Esta omisión impide que la mujer se interese por otras alternativas al aborto, ya que este 
es presentado como eficaz, sencillo y libre de riesgos importantes para su vida y su salud, lo cual es cuestionable científicamente.

- Información sobre el pronóstico en el PAT: la madre gestante debería conocer previamente a la entrega de su CI la evolución de su estado de salud; tanto de la ejecución del aborto, como de la negativa de hacerlo. Al no ser incluido en este documento, también ocurre un sesgo informativo para la mujer.

- Información sobre las alternativas del procedimiento abortivo en el PAT: este documento normativo presenta al aborto terapéutico como una alternativa y regula que la mujer gestante debe conocerla previamente a la entrega de su CI (art. 6.1), sin embargo, en ninguna parte menciona, ni mucho menos detalla, a otras alternativas, situación que hace del aborto la única opción para la mujer, lo cual no es verdadero.

\section{Información contenida en los} formularios de Consentimiento Informado: los formularios de $\mathrm{Cl}$ que el PAT anexa también deben ser analizados, ya que son el soporte físico de este derecho y registran de manera escrita la información relevante para la madre gestante.

En su primer formulario, el PAT recaba la autorización de la madre para el aborto. Sin embargo, no puede entenderse como un modelo base al cual se le deba adaptar con la información que la paciente necesite, pues al igual que los otros anexos, no contiene espacios para la información referente a su estado clínico y los derechos que se deben informar en cada caso concreto. Simplemente contiene un espacio libre de una línea para nombrar la entidad clínica por la cual se aborta. A una observación directa, es un formato acabado que sólo debe llenarse con la firma de la mujer, el nombre de la entidad clínica diagnosticada (enumeradas en el art. 6 inc. 1), la fecha, la firma y huella dactilar de la usuaria, el sello, la firma de la persona que brinda la orientación y consejería, así como el nombre, firma y sello del médico tratante, quien declara procedente el aborto. Esto último, es insuficiente para aclarar la responsabilidad de los involucrados, dado que no figura el médico designado para realizarlo, quien, según el PAT, también debe informar a la mujer y hacerle firmar el formulario de CI.

El formulario en mención igualmente contiene una breve declaración de la madre gestante sobre la recepción verbal de la información sobre los inconvenientes, beneficios y riesgos asociados al aborto. No obstante, en lugar de tratar sobre el riesgo para su vida generado por su embarazo y el mal grave y permanente que le causará a su salud, se refiere a la certeza de que dicho mal le ocurrirá si se negara a proporcionar su CI para el aborto, lo cual no puede aseverarse médicamente; omitiéndose también la posibilidad, por mínima que sea, de que este no ocurra. Consideramos que la omisión de la información escrita en el formulario y la simple declaración de la madre de su recepción verbal le hace carecer de garantías informativas previas a su CI.

Por su parte, el anexo 2 contiene el formulario destinado para los casos de entrega del CI por representación, que ha de consignar la firma del representante de la mujer gestante que sea incapaz, tiene un tenor similar.

El anexo 3 contiene el formulario para la revocatoria, de la paciente o su 
representante, del CI y autorización del procedimiento. Este anexo, tampoco contiene la información escrita y detallada sobre el riesgo de la revocatoria de la decisión de abortar para la vida o la salud de la madre gestante. ${ }^{6}$

\section{Fases de la comunicación y acceso a la información}

La madre gestante debería contar con toda la información relevante en el momento oportuno: antes de tomar alguna decisión sobre proceder con el aborto por razones terapéuticas o no. Veamos si ocurre de esta manera.

\section{El momento de la entrega de} la información: la indicación de proporcionar la información a la madre gestante se encuentra programada para dos momentos en el PAT. El primer momento ocurre después de la primera revisión que el médico tratante le realiza a la mujer gestante. Dicho médico le da el primer diagnóstico sobre el estado de su embarazo y el riesgo que este implica para su vida, o el mal grave y permanente que causa sobre su salud. También recibe la información sobre los procedimientos que correspondan, ${ }^{7}$ después de lo cual la mujer puede solicitarle a su médico tratante que se le practique un aborto por razones terapéuticas.

El segundo momento de entrega de información se da después de la designación del médico designado para realizar el aborto, distinto del médico tratante. El PAT contiene un apartado que trata expresamente sobre el CI (art. 6.6, primer párrafo). En donde establece el deber de información del médico designado, el mismo que "debe asegurarse que la gestante, o en su caso, su representante legal, con ayuda del citado profesional, complete y firme el formulario preestablecido para el consentimiento informado y autorización del procedimiento" (art. 6.6, segundo párrafo).

La madre gestante tiene clara su decisión de abortar desde que le solicita a su médico tratante la realización de un aborto, después de la atención en el primer momento, requeriría de más tiempo, así como de más información para tomar una decisión. Sin embargo, el PAT sólo establece un tiempo máximo de 24 horas para la programación y uno de 06 días para la ejecución del aborto desde que se obtiene el CI de la mujer (art. 6.2.5), no contemplando un tiempo mínimo que garantice el proceso de recepción y comprensión de la información de la paciente, lo cual da lugar a eventuales vulneraciones del proceso de toma de $\mathrm{CI}$, ya que pueden ser objeto de persuasiones por parte del médico tratante para agilizar su trabajo y desempeño.

Lo correcto habría sido que el PAT regulase la entrega de toda la información desde el primer momento, antes de la presentación de la solicitud, a fin de que la madre cuente con todas las herramientas necesarias para consentir o no libremente un aborto. Igualmente, la mujer ya ha tomado la decisión antes de ser atendida en el segundo momento y ya ha brindado su $\mathrm{CI}$, lo que demuestra que el segundo momento consiste en un procedimiento de mero trámite administrativo, que no cumple con la normativa correspondiente al CI.

6 Sólo contiene la siguiente declaración: "... que he recibido información y comprendido lo siguiente: Mi embarazo actual (o el de mi representada) pone en serio riesgo mi (su) vida, o causará un mal grave y permanente en mi (su) salud.

7 Faltando información importante, que recibe después de tomar optar por el aborto: los riesgos en el caso de someterse al aborto, su pronóstico, tampoco las alternativas al aborto a las que hubiere lugar, y toda la información relevante para la toma de una decisión libre y razonada (art. 6 inc. 6). 
Entrega de información y la responsabilidad del médico en el

PAT: en los momentos de la entrega de la información previa al CI regulados por el PAT intervienen expresamente dos médicos: el tratante y el designado para la ejecución del aborto. Sin embargo, hemos advertido que en los formularios que el PAT anexa figuran dos personas: el médico tratante y la persona que brinda la orientación y consejería, quienes imprimen su firma y sello, asumiendo la responsabilidad correspondiente.

No figura en dicho formulario el médico designado después. Lo contradictorio es que, como hemos visto en apartados anteriores, el PAT regula el deber de información previa al CI en la persona del médico designado para el aborto, no así para el médico tratante. La información que proporciona este último es menor e incompleta para la toma de una decisión libre, lo que no ocurre, al menos formalmente, con la información debida que proporciona el médico designado para realizar el aborto. Según esta dinámica, el médico tratante, que es quien firma el CI, sólo responderá por la información que debe proporcionar a la paciente, en un primer momento de la atención, que no se encuentra como un deber propiamente dicho y, como ya lo hemos dicho, es insuficiente, no pudiéndosele exigir la información que debería haber proporcionado el médico que realiza el aborto. Este último, tiene el deber de proporcionar más información, pero no firma el formulario de $\mathrm{CI}$, por lo que no asume la responsabilidad por su entrega insuficiente o nula. Esta dinámica vulnera el derecho al CI de la madre gestante, quien, ante algún reclamo por la falta de información debida, que de haberla recibido no habría consentido del aborto, no podrá exigirle al médico obligado a proporcionársela ninguna clase de responsabilidad.

\section{Servicio de información y} orientación o consejería: La información y orientación o consejería que recibe la mujer debería ser un apoyo para la misma, a fin de colaborar con ella para la toma de decisiones libres e informadas. El PAT trata sobre la orientación y consejería que debe recibir la paciente en todo momento (art. 6 inc. 5). No obstante, delimita el momento de dicha orientación "desde que se decide la intervención hasta después de realizada, a cargo del personal profesional capacitado" (art. 6 inc. 5). No regula que deba darse antes de la toma de la decisión, por lo que los profesionales no emplearán sus conocimientos y capacitación para informarle a la madre gestante sobre su condición de salud y las consecuencias de optar por el aborto, como de continuar con su embarazo antes de su toma de decisión. Asimismo, destacamos que la firma y el sello de la persona que brinda dicha orientación es requerida en el formulario de CI de la madre gestante, algo que es inadecuado, ya que la mujer no recibe la información de algún profesional encargado, este cumple su deber con la mujer recién a partir de la decisión de proceder con el aborto (art. 6 inc. 5), y no antes. Con esto, el PAT se contradice directamente, no siendo cierto que la paciente recibe una consejería en todo momento.

\subsection{Entrega del Consentimiento Informado para la realización de un aborto terapéutico}

$\mathrm{El} \mathrm{CI}$ se encuentra regulado con rango de ley en el art. 4 de la LGS, con el siguiente tenor: "ninguna persona puede ser sometida a tratamiento médico o quirúrgico, sin su consentimiento previo o el de la persona llamada legalmente a darlo, 
si correspondiere o estuviere impedida de hacerlo". Asimismo, se encuentra en el art. 27 del Reglamento de la Ley que establece los Derechos de las Personas Usuarias de los Servicios de Salud: "para aplicar tratamientos especiales, realizar pruebas riesgosas o practicar intervenciones que puedan afectar psíquica o físicamente al paciente, el médico está obligado a obtener por escrito su consentimiento informado". Con la salvedad de que la "La firma del Consentimiento Informado no exime de responsabilidad a los profesionales de la salud... frente a eventos de mala praxis que pudieran producirse en desmedro de la salud de las personas usuarias" (art. 24)

En las líneas sucesivas analizamos la normativa específica aplicable al CI en lo referente a: la manifestación de la voluntad de la madre gestante y la valoración jurídica del formulario de CI para la realización de un aborto por razones terapéuticas.

Manifestación de la voluntad de la madre gestante: el art. 15 inc.4.a de la Ley $\mathrm{N}^{\circ} 29414$ modifica el art. 15 de la LGS, incluyendo expresamente el derecho de toda persona "a otorgar su $\mathrm{CI}$, libre y voluntario, sin que medie ningún mecanismo que vicie su voluntad, para el procedimiento o tratamiento de salud..." Según el Reglamento de esta Ley, la manifestación de la voluntad de la madre gestante debe ser libre. Esto abarca la decisión de iniciar un tratamiento, optar por uno de todos los que se les informe como disponibles, o el de negarse a recibir algún tratamiento, dentro de los límites que establece la Ley, teniendo derecho a los cuidados paliativos disponibles para su enfermedad (Decreto Supremo No 027 2015-SA, 2015). ${ }^{8}$

\section{Defectos de información y vicios} del consentimiento: La manifestación de la voluntad puede verse viciada por el error, el dolo, la violencia o la intimación al momento de proporcionarla, dichos vicios pueden generar la anulación del acto jurídico (Código Civil. D. Leg. 295, 1984, Arts. 201 - 216), lo cual genera que pierda su eficacia y, por lo tanto, no haya obligación para las partes comprometidas.

La teoría del CI contempla tres situaciones que vician el consentimiento: la persuasión, la coacción y la manipulación, ya que impiden que el paciente pueda manifestar su voluntad libremente, para consentir sobre las terapias que se le propongan (Pérez Dávila, 2017, pp. 45 47). El art. 23 del Reglamento de la Ley $N^{\circ} 29414$, es coherente con esta doctrina, ya que no admite ningún mecanismo que distorsione o vicie la voluntad del paciente y regula que "ello se constituirá en circunstancia que acarrea la nulidad del acto del consentimiento para el procedimiento o tratamiento de salud".

El Código Civil (CC) establece que la omisión (art. 212) de la entrega de dicha información puede darse por un dolo causante, cuando "el engaño usado por una de las partes haya sido tal que sin él la otra parte no hubiera celebrado el acto" (art. 210), o por dolo incidental, cuando el engaño no haya determinado la voluntad del acto (art. 211). La falta de información que permite el PAT es de carácter importante, ya que sólo considera, parcialmente, aquella referida al acto abortivo, presentándoselo a la madre gestante como una acción indispensable para salvar su vida, o evitar en su salud un mal grave y permanente. Al omitir referirse a la enfermedad de la

8 Asimismo, este Reglamento regula el derecho al respeto del proceso natural de la muerte del enfermo terminal y expresa la posibilidad de sufrir las acciones punibles del Código Penal ante la acción u omisión contraria a dicho respeto (Art. 23). 
mujer gestante, a las alternativas con las que podría contar, y a los riesgos de optar por el aborto, o de continuar con el embarazo, la mujer no tiene garantías informativas para recibir todos los datos y tomar una decisión libre. Estas omisiones afectan en la toma de decisión de la mujer, por lo que, ante esta circunstancia, nos encontraríamos ante el dolo de tipo "causante".

Por otra parte, la mujer también podría ver viciado su CI por la existencia de un error, el mismo que se da cuando las partes del acto jurídico consideran como verdadero lo que es falso, o como falso lo que es verdadero, y es distinguible de la ignorancia, debido a que esta se caracteriza por la falta de conocimiento y no por la deformidad de la realidad (Flores, 2002, p. 309). En el caso del aborto terapéutico, dicho error se presenta cuando el médico sólo entrega la información normada por el PAT, considerando que este limita su responsabilidad informativa para con la mujer. Debido a que el médico puede darse cuenta del deber de información plena que tiene para con la mujer, ya que forma parte de la práctica del CI, cotidiana para los diversos procedimientos clínicos que debe llevar a cabo como parte de la lex artis de su profesión, nos encontramos ante un error conocible (art. 203 CC). Asimismo, al ser un error que "recae sobre la propia esencia o una cualidad del objeto del acto", es determinante de la voluntad (art. 202 CC), la falta de información completa previa al CI, constituye un error esencial. Ya que, por ejemplo, la mujer que hubiese contado con la información oportuna sobre las alternativas terapéuticas que tiene el aborto, no consentiría en la eliminación de su hijo de una manera directa.

Defectos de información y responsabilidad civil: La anulación del acto jurídico por dolo o por error opera con efectos retroactivos al momento de su celebración (art. 222), lo que implica la devolución de las prestaciones (por ej. de la cosa y del dinero en el caso de una compraventa). Esto no es posible para el caso de la madre gestante que proporcionara su CI y se realizara un aborto sin haber recibido la información completa y que, después del mismo, tomara conocimiento de la falta de dicha información, ya que la vida de su hijo es imposible de restituir. Por esto, resulta importante hablar de la responsabilidad del médico que omite proporcionar la información debida.

El CC establece que el profesional sólo responde por los daños y perjuicios generados durante la prestación de sus servicios ocasionados mediante el dolo o la culpa inexcusable, cuando la realización del servicio requiera la solución de problemas de especial dificultad (art. 1762). Dicho profesional, tendría la obligación de indemnizar por daños y perjuicios, debido a su omisión de cumplir con sus obligaciones por dolo, o culpa inexcusable (art. 1321). En este sentido, la mujer tendría derecho a solicitar una indemnización sólo en los casos en que el médico omite brindar la información mediando el dolo, no así el error, del que hemos hablado antes, por ser de aplicación el art. 207 CC, que establece la improcedencia de la indemnización ante la anulación del acto jurídico por error.

El PAT orienta al profesional de la salud hacia la culpa inexcusable en el momento de la entrega de la información, quien debería considerar al CI como parte de la lex artis de su profesión. Así, al no ejecutar la obligación de brindar dicha información cometería una negligencia grave (art. 1319 CC), que le permitiría a la mujer afectada exigir una indemnización a causa de un daño emergente (art. 1321 CC) y daño moral (art. 1322 CC), según sea el caso. 
Valoración jurídica del formulario de Consentimiento Informado: teniendo en cuenta que la forma mediante la cual se realiza el acto jurídico es uno de sus requisitos de su validez "bajo sanción de nulidad" (art. 140 inc. 4 CC). En esta parte, nos proponemos determinar si el formulario de CI que debe firmar la madre gestante constituye un requisito de validez del mismo, o si es un medio de prueba.

Para que una forma sea considerada de cumplimiento obligatorio, no basta con la indicación de una forma determinada por la Ley, muchas veces escrita, sino que dicha Ley también debe indicar la sanción de nulidad. Cuando la Ley no impone la sanción de nulidad, la pérdida o destrucción del documento permite la prueba del acto a través de otro medio probatorio, ya que tiene una función procesal y no sustantiva, por ser un medio probatorio y no un elemento necesario para la validez del acto (Cortez, 2017, pp. 212 213).

En concordancia con el art. 27 LGS, la Ley $N^{\circ} 29414$, que modifica el art. 15 de la LGS sobre los aspectos específicos de la información previa y el CI de los pacientes, regula que este debe realizarse bajo la forma escrita y en un documento oficial que permita ver el proceso de información y decisión, con la firma del paciente, o, si no supiese hacerla, con su huella digital (art. 15 inc. 4. a.2) para los casos siguientes casos: a) intervenciones quirúrgicas, b) anticoncepción quirúrgica, c) procedimientos que puedan afectar la integridad de la persona. De la misma forma se encuentra regulado en el art 23 de su Reglamento, y establece la obligatoriedad para las situaciones antes mencionadas. Pese a que establece la forma escrita, la Ley no consigna en su redacción la sanción de nulidad, por lo que podemos concluir que la forma establecida para el CI en el Perú es un medio probatorio y no un elemento constitutivo del mismo.

El aborto terapéutico está inmerso en las circunstancias que obligan a la entrega de un $\mathrm{Cl}$ escrito. Dado que consiste en una intervención invasiva, ya sea mediante la aspiración manual endouterina, el uso de misoprostol, o el legrado uterino (art. 6 inc. 7 PAT), con consecuencias relevantes para la salud de la madre gestante, y la persona en formación intrauterina, el $\mathrm{CI}$ para su realización ha de ser explícito y bajo la forma escrita. Por este motivo, el PAT contiene un apartado específico sobre el $\mathrm{CI}$ de la madre gestante (art. 6 inc. 6), en donde se puntualiza sobre la firma del formulario preestablecido para el CI y la autorización del procedimiento. En este sentido, el PAT está en coherencia con la Ley antes mencionada.

No obstante, ello, la Ley también regula que el formulario debe permitir ver el proceso de información y decisión, cuestión que no se encuentra detallada por el PAT en su formulario; razón por la cual, el PAT contraviene la Ley.

El formulario y la carga de la prueba: como en el PAT los formularios son genéricos, no personalizados y contienen información incompleta, no cuentan con los requisitos indispensables para el CI y terminan siendo declaraciones de exclusión de responsabilidad del profesional. Razón por la cual no constituyen una prueba absoluta de una decisión libre y consciente, son, por tanto, susceptibles de ser revisados judicialmente e, incluso desestimados como prueba (García, 2015, pp. $17-18$ ).

De otro lado, dicho formulario prueba que no se proporcionó la información 
escrita, ya que esta no figura; no obstante, por sí mismo no llega a probar la omisión de la explicación médica verbal y completa a la mujer. Recordando que el CI es un proceso, no se podría desestimar la diligencia del médico durante el trato personal, que de haber existido, se podría afirmar que hubo un CI válido para el aborto terapéutico.

Por esto, es preciso determinar la persona sobre la cual recae el deber de probar. Según el art. 1330 CC: "La prueba del dolo o de la culpa inexcusable corresponde al perjudicado por la inejecución de la obligación, o por su cumplimiento parcial, tardío o defectuoso". Por esto, la mujer que aborta por razones terapéuticas guiada por una información insuficiente debe probar el dolo o la culpa del médico al no informarle debidamente. Esto le genera una desventaja, toda vez que es el médico y la institución para la cual este labora profesionalmente quienes tienen la historia clínica de la paciente, por lo que poseen más herramientas de prueba, ${ }^{9}$ generando una suerte de prueba diabólica (Velazco, 2013, pp. 675 - 680).

\section{CONCLUSIONES}

El CI posee distintos enfoques, según la bioética desde la cual se comprenda. Desde la Bioética personalista, forma parte de la lex artis de la medicina. Es un proceso que se desarrolla a lo largo de la relación médico - paciente, en donde ambos se deben valores de fidelidad mutua. El paciente es el principal responsable de su vida y de su salud, y el médico es su colaborador. Desde la bioética principialista, dicho Consentimiento surge de una relación médico- paciente de carácter mercantil. El primero, otorga sus servicios profesionales a cambio de una retribución económica, y el segundo se encuentra interesado en recuperar su salud, por lo que paga la cantidad de dinero correspondiente.

El CI regulado en el PAT se orienta hacia la bioética principialista. En primer lugar, porque sólo la madre gestante, quien posee la capacidad para vincularse contractualmente, es la persona con la que se desarrolla la relación médico paciente, no así su hijo en desarrollo. La misma que, a su vez, es considerada como tal sólo después de la firma del formulario de CI y no durante toda la atención médica. En segundo lugar, porque el $\mathrm{CI}$ se da como resultado de la solicitud de la madre gestante para el aborto terapéutico, sobresaliendo su autonomía. Y, en tercer lugar, porque en aplicación del principio de "beneficencia", el CI de la mujer no causa daño a terceros y, al contrario, repercute en un beneficio para la sociedad, que con el aborto disminuye los gastos que destinaría a la atención integral de la gestante a lo largo de su complicado embarazo.

El PAT sólo nombra los aspectos informativos que la mujer debe recibir para su CI, omitiendo detallarlos individualmente tanto a lo largo de su redacción, como en los formularios de toma de CI que anexa, dando lugar a que se vulnere su derecho a consentir libremente. Dichos aspectos, le son proporcionados en dos momentos. En el primeo, toma conocimiento sobre su embarazo, y no sobre su estado complicado de salud. Después de lo cual debe decidir sobre solicitar un aborto por indicación terapéutica o continuar con su embarazo. En un segundo momento,

9 El art. 23 del Decreto Supremo № 027-2015-SA norma que el proceso de CI "formará parte de la historia clínica del usuario, siendo responsabilidad de la IPRESS su gestión, custodia y archivo correspondientes". 
debe recibir la información referente al procedimiento abortivo, incluyendo los riesgos y las alternativas al mismo, para proseguir con la firma del formulario de CI.

Los formularios de Consentimiento Informado, anexados en el Protocolo de Aborto Terapéutico, sólo poseen un valor probatorio, no son un elemento constitutivo del CI. Sitúan a la madre gestante ante una posición desventajosa en el caso de que haya consentido el realizarse un aborto sin haber recibido la información previa verbalmente y deba probar los hechos, ya que no cuenta con la documentación necesaria, con la que sí cuenta el médico.

El incumplimiento de la información previa al Consentimiento Informado de la madre gestante genera vicios en la manifestación de su voluntad. Puede ocasionarse por un "dolo causante" del médico, quien, a través de la persuasión y la manipulación, sirviéndose de la falta de garantías de la información del Protocolo en cuestión, induzca a la madre gestante a la realización de un aborto evitable. Asimismo, puede estar viciada por el "error esencial y conocible" del médico, que, limitándose al cumplimiento del Protocolo, omita la entrega de la información relevante para la toma de una decisión consciente.

La indemnización por los daños generados por la falta de información completa, detallada y previa al CI de la madre gestante sólo tiene lugar cuando mediare el dolo, o la cumpla inexcusable del médico.

\section{REFERENCIAS}

Acosta, José. (2011) "La ética clínica cubana: ¿paternalismo, principalismo, personalismo o electivismo?". En Revista Redbioétical UNESCO., Número 3.

Aguilar, L. (2008) "Consentimiento informado en la paciente embarazada menor de edad". En Revista mexicana de anestesiología, Volumen 31, Suplemento I.

Álvarez, J. (2008) "El concepto de interrupción voluntaria del embarazo (IVE) en bioética". En Revista de la Facultad de Medicina UNAM, Volumen 51, Número 6.

Bernar, A. (2000) Valoración moral del consentimiento informado como expresión de la relación médico-paciente. Tesis para optar el grado de Doctor, Pontificia Universidad de la Santa Cruz. Roma.

Besio, M. (2013) "La mujer gestante y su hijo. El médico frente al llamado aborto terapéutico". En Aborto y anticoncepción de emergencia: aspectos antropológicos, éticos y jurídicos. Ecuador: EDILOJA.

Bessio, M, Chomalí, F, et al. NEIRA, Jorge, et al. (2008) Aborto "terapéutico": consideraciones médicas, éticas, jurídicas y del magisterio de la Iglesia Católica. Chile: Pontificia Universidad Católica de Chile

Burgos, J. (2013) "¿Qué es la bioética personalista? Un análisis de su especificidad y de sus fundamentos teóricos". En Cuadernos de Bioética., Volumen 24, Número 1.

Carrasco, I. (2005) "El concepto de persona y su relevancia axiológica: los principios de la bioética personalista". En Revista internacional de bioética, deontología y ética médica, Número 3, Volumen 16. 
Chirinos, E y Chirinos, F. (2006) La Constitución: lectura y comentario. 5ta ed. Perú: Rodhas.

Civeira, E. (2010) "Consentimiento por representación: cuestiones problemáticas en Medicina Crítica". En Cuadernos de la Fundación Víctor Grífols i Lucas, Número 22.

Cortez, c. (s.a) La forma del acto jurídico en el Código Civil peruano de 1984. Recuperado de:file:///C:/ Users/Usuario/Downloads/DialnetL aFormaDelActoJuridicoEnElCodigo Civil PeruanoDe1984-4133684.pdf

Engelhardt, H. (1995) Los fundamentos de la Bioética, traducido por Arias, I, 2da ed. Barcelona: Paidós Ibérica.

Feito, L. (2011) "Fundamentos de bioética, de Diego Gracia". En Bioética y debat, Número 17.

Ferro, M et al. (2009) "La bioética y sus principios". En Acta Odontológica Venezolana, Número 2, Volumen 47.

Flores, P. (2002) Diccionario jurídico jundamental. 2da ed. Lima: Grijley.

Fuentes, M. (2006) Principios fundamentales de bioética, Argentina: Instituto del Verbo Encarnado.

García, J. (2015) "La responsabilidad civil médica en el Perú: aspectos básicos". En Derecho y Cambio Social, noviembre.

García, J. (2013) "Bioética personalista y bioética principialista. Perspectivas". En Cuadernos de Bioética XXIV, Número 7.

Gómez, P. (2009) "Principios básicos de bioética". En Revista Peruana de Ginecología y Obstetricia, Número55.

Gómez-Fajardo, C. (2014) "Observaciones bioéticas sobre el "Consenso de Estambul": el cientificismo y la omisión de lo humano en la embriología humana". En Persona y bioética, Número 1, Volúmen 18.

Gonorazky, S. (2015) "Los principios éticos universales y su aplicación a los ensayos clínicos de medicamentos". En Revista Patagónica de Bioética. Año 1, Número 2.

Herrera, F. (1999) El derecho a la vida y el aborto. 2da. ed. Bogotá: Universidad del Rosario.

López Moratalla, N. (2010) "El cigoto de nuestra especie es cuerpo humano". En Persona y bioética., Número 2, Volumen 14.

López, N. (2012) "¿Cómo cambia un aborto el cerebro?". En Cuadernos de Bioética, Número 23.

Lucas, R. (2005) Explícame la bioética: guía explicativa de los temas más controvertidos sobre la vida humana. Madrid: Palabra.

Martín, I. (2005) Bioética, religión y salud. Madrid: Subdirección general de bioética y orientación sanitaria.

Novoa, R. (2013) Nivel de conocimiento sobre el consentimiento informado en la práctica médica en internos de medicina en el Hospital Nacional Dos de Mayo. Tesis para optar el Título de Médico Cirujano, Universidad Nacional Mayor de San Marcos. Lima. 
Ossadón, M. (2012) "Aborto y justificación". En Revista Chilena de Derecho., Número 2, Volumen 39.

Pardo, A y Echarte, L. (2015) "La formalización del consentimiento informado en investigación y la pérdida sentimental del paciente". En Persona y Bioética., Número 2, Volumen 19

Pardo, A. (2008) "Sobre el acto humano: aproximación y propuesta", Revista científica Persona y bioética, 2 (31), pp. 78-107.

Pardo, A. (2012) La ética de la bioética: valores, principios y normas, Recuperado de: www. unav.es/humbiomedicas /apardo/ eticabioetica.pdf

Penchaszadeh, V. (20 1 5) "Perspectivas de la bioética en Latinoamérica y el caribe". En Revista Patagónica de Bioética., Número 2.

Pérez Dávila, B. (2017) Implicancias ético-legislativas del Consentimiento Informado en la redacción del Protocolo de Aborto Terapéutico para la madre gestante. Tesis para optar el grado de Maestro en Persona, Matrimonio y Familia, Universidad Católica Santo Toribio de Mogrovejo. Lima.

Pérez, J. (2009) "El bien de la vida y el bien de la salud: el deber de preservarlos". En Junto al enfermo incurable $y$ al que muere: orientaciones éticas y operativas. Madrid: Pontificia Academia Pro-Vita.

Prieto, S; Sainz, A. (2010) Consentimiento informado y otros aspectos bioéticos de la información al paciente en el ámbito del laboratorio clínico. Recuperado de: http://www.aebm.org/grupos\%20 de\%20trabajo/docu2.pdf

Requena, P. (2005) El principialismo y la casuística como modelos de la bioética clínica. Tesis para optar el grado de Doctor, Pontificia Universidad de la Santa Cruz. Roma.

Rubio J y Del Trigo, M. (2005) "Consentimiento Informado" en Bioética y Derechos Humanos: Implicaciones Sociales y Jurídicas. Sevilla: Universidad de Sevilla.

San Martín, J. (2015) Antropología filosófica II: vida humana, persona y cultura. Madrid: Universidad Nacional de Educación a Distancia.

Serrano, J (1993) Bioética, poder $y$ derecho. Madrid: Universidad complutense,

Sgreccia, E. (2007) Manual de Bioética, Tomo I, 4ta ed. Madrid: Biblioteca de autores cristianos.

Suardíaz, J. (2006) "Aportes y limitaciones del principialismo en bioética". En Revista del Centro de Bioética Juan Pablo II. Número 1, Volumen 6.

Velazco, C. (2013) Regulación jurídica del deber de información previa al consentimiento informado en el aborto. Tesis para optar el grado de Doctor, Universidad Complutense de Madrid. Madrid.

Vizcaya, D. et al. (2014) "Conocimiento de los pacientes sobre el consentimiento". En Revista de la Facultad de Medicina de 
la UNAM, Número 5, Volumen 57.

\section{NORMAS}

Código Civil. (1984). D. Leg. 295. Perú.

Código Penal. (1991). D. Leg. 635. Perú.

Guía Técnica de Gestión de Promoción de la Salud en Instituciones Educativas para el Desarrollo Sostenible. (2011). Resolución Ministerial $N^{\circ} 298$ 2011/MINSA. Perú.

Guía Técnica de Gestión de Promoción de la Salud en Instituciones Educativas para el Desarrollo Sostenible. (2010). Resolución Ministerial $N^{\circ} 1021$ 2010/MINSA. Perú.

Guía Técnica Nacional para la estandarización del procedimiento de la Atención Integral de la gestante en la Interrupción Voluntaria por indicación Terapéutica del Embarazo menor de 22 semanas con consentimiento informado en el marco de lo dispuesto en el artículo $119^{\circ}$ del Código Pena. (2014). Resolución Ministerial $N^{\circ}$ 486-2014/MINSA. Perú.

Guía Técnica para la Evaluación de la Satisfacción del Usuario Externo en los Establecimientos y Servicios Médicos de Apoyo. (2011). Resolución Ministerial $N^{\circ}$ 527-201 1/MINSA. Perú.

Ley General de Salud. (1997). Ley N²6842. Perú.

Ley que establece los Derechos de las Personas Usuarias de los Servicios de Salud. (2009). Ley $N^{\circ}$ 29414. Perú.

Reglamento de la Ley que establece los Derechos de las Personas Usuarias de los Servicios de Salud. (2015). Decreto Supremo No 0272015-SA. Perú. 